\title{
Conocimiento de las normas de tráfico en función de la edad y de la importancia para la seguridad vial
}

\author{
Knowledge of Traffic Rules According to the Age \\ and the Importance for Road Safety
}

Recibido: septiembre 12 de 2011 | Revisado: abril 9 de 2012 | Aceptado: junio 20 de 2012

\author{
CRISTINA VARGAS* \\ CÁNDIDA CASTRO ** \\ Francisco JaVIER MARTOS $* * *$ \\ Humberto Manuel TrujILlO **** \\ Universidad de Granada, España
}

\begin{abstract}
RES UMEN
Un déficit en la memoria producido por la edad puede influir en el conocimiento de la normativa de tráfico. El presente estudio analiza las diferencias en el conocimiento de la normativa en función de la edad e importancia para la seguridad vial. Participaron 300 personas (18-75 años). Se elaboró un cuestionario de conocimiento sobre normativa de tráfico, según la importancia que desempeñan para la seguridad vial (poco importantes, importantes y muy importantes). En general, el conocimiento de los conductores sobre la normativa de tráfico fue pobre. Contrario a lo esperado, el rendimiento de los conductores mayores no siempre fue inferior al de los otros grupos de edad, presentando mejores resultados en preguntas poco importantes y muy importantes.

Palabras clave autores

Conductores mayores, edad, conocimiento de las normas de tráfico, seguridad vial. Palabras clave descriptores

Psicología del Tráfico y de la Seguridad, España, investigación cuantitativa.
\end{abstract}

tos, F. J. \& Trujillo, H. M. (2012). Conocimiento de las normas de tráfico en función de la edad y de la importancia para la seguridad vial. Universitas Psychologica, 11(4), 1277-1289.

* Docente-Investigador. Departamento de Metodología de las Ciencias del Comportamiento. Facultad de Psicología. E-mail: cvargas@ugr.es

** Docente-Investigador. Departamento de Psicología Experimental. Facultad de Psicología. E-mail: candida@ugr.es

***** Docente-Investigador. Departamento de Psicología Experimental. Facultad de Psicología. E-mail: fmartos@ugr.es. ResearcherID: Martos, F. J. A-6909-2013.

****** Docente-Investigador. Departamento de Metodología de las Ciencias del Comportamiento. Facultad de Psicología.E-mail: humberto@ugr.es

\begin{abstract}
A B S T R A C T
Age-associated memory impairment could worsen the knowledge of traffic rules. The present study analyses the differences in the knowledge of the traffic rules according to the age and the importance for road safety. 300 drivers participated (18-75 years-old). A questionnaire was developed to assess the knowledge of the traffic rules according to the importance for road safety (less important, important, and more important questions). Generally, the knowledge of the traffic rules was poor among drivers of all ages. Older drivers' scores were not always lower than the younger and adult drivers. These results are a little surprising and not the outcome we originally predicted, they show the older drivers had far better results in the less important and more important questions.

Key words authors

Older Drivers, Age, Knowledge of Traffic Rules, Road Safety.

Key words plus

Traffic Psychology, Traffic Security, Spain, Quantitative Research.
\end{abstract}


La población de los países industrializados está envejeciendo. Por ejemplo, España ha experimentado uno de los procesos de envejecimiento más rápidos del mundo donde el 16.7 \% de la población está constituido por personas de 65 o más años (Instituto de Mayores y Servicios Sociales, 2009). En consecuencia, el número de conductores mayores se ha incrementado sensiblemente, pues el automóvil es una herramienta básica para mantener una calidad de vida adecuada en este grupo de edad, al proporcionales autonomía y movilidad (Charlton et al., 2006; Simões \& Marin-Lamellet, 2002). Garantizar la seguridad de estos conductores es un objetivo prioritario, pues las habilidades necesarias para el desarrollo de una conducción segura se ven afectadas con la edad (Eby, Trombley, Molnar \& Shope, 1998; Marmeleira, Godinho \& Vogelaere, 2009). Este deterioro producido por el envejecimiento parece estar en la base de un incremento en el riesgo de sufrir accidentes y en la vulnerabilidad a los mismos, siendo la probabilidad de fallecer superior en los conductores mayores en comparación con los jóvenes (Bédard, Guyatt, Stones \& Hirdes, 2002; Charlton et al., 2006; Hakamies-Blomqvist, 1998; Insurance Institute for Highway Safety, 2001; Li, Braver \& Chen, 2003; Oxley et al., 2005; Simões $\&$ Marin-Lamellet, 2002).

Entre las habilidades necesarias en la conducción que disminuyen con la edad se encuentran la percepción visual, las habilidades psicomotoras y distintos factores cognitivos (Barrash et al., 2010; Campagne, Pebayle \& Muzet, 2004; Lundberg, Hakamies-Blomqvist, Almkvist \& Johansson, 1998; Mathias \& Lucas, 2009; Prada, Pineda, Mejía \& Conde, 2010). El deterioro de la memoria operativa y de la episódica es uno de los cambios cognitivos más relevantes que se producen con la edad (Simões \& Marin-Lamellet, 2002). Estos déficits dificultan la capacidad de los conductores mayores para afrontar tareas diarias como la conducción, especialmente en ambientes dinámicos donde se requiere actualizar frecuentemente las representaciones mentales de la situación (por ejemplo, al conducir en carreteras desconocidas o en momentos del día con mucho tráfico). El deterioro de la memoria no influye tanto si el escenario de conducción es familiar. En cambio, conducir en situaciones donde aparezcan aspectos novedosos de la normativa de tráfico y/o el escenario no sea familiar podría dificultar una conducción segura en los conductores mayores (Caserta \& Abrams, 2007).

Los déficits en la memoria pueden influir negativamente en el reconocimiento de la normativa de tráfico que estaba vigente cuando el conductor obtuvo inicialmente el permiso de conducir. Además, éstos también pueden dificultar la adquisición y el recuerdo de los cambios normativos que se hayan producido durante años posteriores a la obtención del permiso (pensemos, por ejemplo, en algunas medidas recientes en España, como la introducción del sistema de permiso por puntos o las modificaciones del Código Penal en materia de Seguridad Vial). En general, estos cambios pueden no ser asimilados adecuadamente por el conjunto de conductores, si no van acompañados de acciones divulgativas eficaces. En el caso de los conductores mayores, la asimilación de los cambios normativos podría ser especialmente problemática como consecuencia de sus déficits de memoria.

De acuerdo con la evidencia anterior sobre el deterioro cognitivo asociado a la edad, se podría hipotetizar que el conocimiento de las normas de tráfico debería ser menor en el grupo de conductores mayores en comparación con otros grupos de edad, lo que tan solo se ha podido comprobar parcialmente en algunos estudios previos. BuelaCasal y Miró (1994) evaluaron mediante un breve cuestionario de 10 ítems el conocimiento de las normas de tráfico y encontraron que, en promedio, los conductores españoles apenas conocían el $48 \%$ de los aspectos más importantes de la normativa. Además, estos autores hallaron una relación negativa entre la antigüedad del permiso de conducir (una variable especialmente relacionada con la edad) y el conocimiento de la normativa. El $52 \%$ de los conductores con una antigüedad de hasta diez años mostraban un conocimiento medio, descendiendo este porcentaje al $48 \%$ de conductores cuando la antigüedad oscilaba entre 11 y 15 años, y a solo el $43 \%$ de los conductores entre 16 y 20 años de antigüedad. Sin embargo, este valor que aumentaba a un $48 \%$ cuando la antigüedad de los 
conductores era superior a 20 años, lo que los autores interpretaron como una mayor presencia de conductores profesionales en este grupo de edad. A este respecto, es importante destacar que el estudio de Buela-Casal y Miró (1994) considera a todos los conductores de más de 20 años de antigüedad en la misma categoría, lo que no permite separar adecuadamente el conocimiento de la normativa de conductores de mediana edad y de mayor edad. Además, el número de preguntas de la normativa de tráfico que se hicieron en este estudio era muy limitado (apenas 10 ítems), no quedando claro el procedimiento utilizado para seleccionarlas y para estimar su importancia.

En otros estudios realizados sobre el conocimiento que los conductores españoles tienen de la normativa de tráfico, se ha encontrado que ignoraban algunas cuestiones importantes sobre el sistema de permiso y licencia de conducción por puntos, un año después de entrar en vigor. Concretamente, el $60.77 \%$ de los encuestados no alcanzaron los 5 puntos (sobre 10) en una prueba de conocimientos sobre el permiso por puntos, siendo la media global de 4.32 puntos (Montoro \& Roca, 2007; Montoro, Roca \& Tortosa, 2008). Por otro lado, en un estudio de Montoro, Roca y Lucas (2008) se observó que el conocimiento que los conductores españoles tienen de los límites de velocidad genéricos es desigual para los distintos tipos de vía. Los porcentajes de mayor desconocimiento se hallaron para las carreteras convencionales (el $54.86 \%$ en aquellas cuyo límite es de $100 \mathrm{~km} / \mathrm{h}$ y el $62.17 \%$ en aquellas cuyo límite es de $90 \mathrm{~km} / \mathrm{h}$ ) y zonas urbanas (32.01\%). En cambio, la velocidad máxima genérica permitida en autopista o autovía fue la mejor conocida por los conductores, respondiendo correctamente un $87.99 \%$ de los encuestados. Sin embargo, estos estudios no analizaron las diferencias en función de la edad, por lo que no permiten valorar si los conductores mayores tienen un mejor o peor conocimiento del permiso por puntos o de los límites de velocidad.

El principal objetivo de la presente investigación es analizar el efecto de la edad en el conocimiento de la normativa de tráfico. En general, se espera que el conocimiento en el conjunto de conductores sea bajo o moderado, tal como sugieren diferentes estudios (Montoro \& Roca, 2007; Montoro, Roca \& Lucas, 2008; Montoro, Roca \& Tortosa, 2008). Por otro lado, los estudios previos sobre el deterioro cognitivo asociado a la edad sugiere un peor conocimiento a medida que aumentan los años. Sin embargo, la evidencia revisada en el ámbito del tráfico no permite generalizar esta tendencia a los conductores de mayor edad respecto a los de mediana edad y, además, existen datos que serían inconsistentes con esta hipótesis (por ejemplo, los conductores con más de 20 años de experiencia mostraron un mejor recuerdo que otros conductores más jóvenes en el estudio de Buela-Casal \& Miró, 1994), por lo que no sería adecuado afirmar que los conductores mayores presentan siempre un peor conocimiento de las normas.

En consecuencia, el presente estudio analiza las diferencias en el conocimiento de la normativa en grupos de conductores jóvenes, adultos y mayores, examinando un conjunto de normas más amplio que en estudios anteriores y utilizando una metodología más objetiva en la selección de los ítems y en la valoración de su importancia. En relación con este último punto, el presente estudio también explora si hay un efecto diferencial en el conocimiento de las normas de tráfico en función de la importancia que representan para la seguridad de la conducción.

\section{Método}

\section{Participantes}

La muestra está constituida por 300 conductores españoles de edades comprendidas entre los 18 y los 75 años. Se han considerado tres grupos de 100 conductores: jóvenes $(M=23.99$ años y $D E=3.69)$, adultos $(M=38.67$ y $D E=5.49)$ y mayores $(M=60.19$ y $D E=6.43)$. El $58.2 \%$ de la muestra son hombres. Se ha excluido una persona del grupo de mujeres mayores por insuficiencia de datos. Todos los participantes debían poseer el permiso de conducir. 


\section{Instrumento de medida}

Para desarrollar el presente estudio se elaboró un cuestionario de conocimientos sobre la normativa de tráfico en España que se componía inicialmente de un total de 30 preguntas dirigidas a examinar el conocimiento de la Ley sobre Tráfico, Circulación de Vehículos a Motor y Seguridad Vial. Para construir este instrumento, en primer lugar, se seleccionaron aleatoriamente 154 preguntas de los tests que la Dirección General de Tráfico (DGT) publica periódicamente en la revista Tráfico. De acuerdo con la DGT, estas preguntas son habitualmente utilizadas para evaluar el conocimiento teórico acerca de la Ley sobre Tráfico, Circulación de Vehículos a Motor y Seguridad Vial, dentro del procedimiento oficial para la obtención del permiso de conducir.

A continuación, un grupo de seis expertos en el área del tráfico y la seguridad vial (tres policías locales y tres guardias civiles de tráfico) valoraron las 154 preguntas según la importancia que desempeñan en la seguridad de la conducción, utilizando para ello las categorías: poco importantes, importantes y muy importantes. Los ítems con un nivel de acuerdo interjueces (evaluado como un porcentaje) inferior al $70 \%$ fueron excluidos del cuestionario. Posteriormente, se promedió la valoración de la importancia que los seis jueces atribuyeron a cada pregunta seleccionada. A partir de esta puntuación de importancia, se escogieron los extremos de 10 preguntas con mayor y menor puntuación, y las 10 preguntas con valores centrales. Con esto se distinguieron tres grupos de 10 preguntas: poco importantes (extremo inferior), importantes (valores centrales) y muy importantes (extremo superior) (Apéndice 1).

Una vez se disponía de esta versión inicial del cuestionario, se aplicó a la muestra de acuerdo con el procedimiento descrito más adelante y se analizó la consistencia interna de los tres grupos de preguntas: poco importantes (alfa de Cronbach $=$ 0.543), importantes (alfa de Cronbach $=0.732$ ) y muy importantes (alfa de Cronbach $=0.725$ ). En el grupo de preguntas poco importantes, que no alcanzaba el criterio de referencia (0.7), se encontró que los ítems 1, 5 y 9 tenían correlaciones negativas con el total, por lo que se procedió a eliminarlos, para mejorar la consistencia interna del grupo de preguntas. Entonces, se volvió a hallar el alfa de Cronbach con los 7 ítems restantes y se obtuvo un valor de 0.716 . A continuación, la variable fue transformada a una escala de 0 a 10 para facilitar la comparación con los otros dos grupos de preguntas (importantes y muy importantes). En consecuencia, la versión final del cuestionario incluye un total de 27 ítems en 3 grupos de importancia (7 poco importantes, 10 importantes y 10 muy importantes).

\section{Procedimiento}

Los cuestionarios fueron administrados en diversos lugares para acceder a la muestra de conductores de distintas edades: a) conductores mayores: en el Aula Permanente de la Universidad de Granada, en las asociaciones de mayores ALUMA y OFECUM, y en los centros de día de San Idelfonso y la $\mathrm{Paz}$ del Patronato de Granada; b) conductores adultos: personal de la Universidad de Granada (administrativos, docentes e investigadores.) y c) conductores jóvenes: estudiantes de la Facultad de Psicología de la Universidad de Granada. Los participantes cumplimentaron los cuestionarios de forma voluntaria e individual, respetando su anonimato. El orden en el que los participantes encontraban los distintos grupos de preguntas (poco importantes, importantes y muy importantes) fue contrabalanceado.

\section{Análisis de datos}

Se realizó un ANOVA factorial con un factor entre-grupos (edad: jóvenes, adultos y mayores) y otro intrasujetos (importancia de la pregunta: poco importantes, importantes y muy importantes). La variable dependiente es el promedio de respuestas correctas obtenidas en el cuestionario. Cuando no se cumplía el supuesto de esfericidad, se ha usado la aproximación épsilon de Greenhouse-Geisser. Las comparaciones a posteriori se han contrastado mediante el método de Bonferroni. Se ha utilizado el paquete estadístico SPSS 15.0.1 (2006) para analizar los datos. 


\section{Resultados}

Se obtuvieron los siguientes resultados (Figura 1). El efecto principal del factor de importancia de la pregunta fue estadísticamente significativo, $F_{\text {Greenhouse-Geisser }}(1.734,508.011)=72.481, p=0.001$, $\eta^{2}=0.198$, al igual que la interacción entre edad e importancia de la normativa, $F_{\text {Greenhouse-Geisser }}(3.468$, 508.011) $=23.423, p=0.001, \eta^{2}=0.138$. El efecto principal de la variable edad no resultó estadísticamente significativo.

Se llevaron a cabo comparaciones a posteriori para examinar el efecto principal de la variable importancia de la pregunta y se obtuvieron las siguientes diferencias significativas: a) entre las preguntas poco importantes $(M=3.78)$ y las importantes $(M=5.21)$, Bonferroni $=-2.442, p=0.001$; b) entre las preguntas poco importantes $(M=3.78)$ y las muy importantes $(M=3.32)$, Bonferroni $=-0.550, p=0.006 \mathrm{y}$ c) entre las preguntas importantes $(M=5.21)$ y las muy importantes $(M=3.32)$, Bonferroni $=1.893, p=0.001$.
La interacción entre la edad y la importancia de la pregunta se examinó mediante el análisis de los efectos simples de la variable edad, de especial interés para los objetivos de este estudio. Se encontraron diferencias estadísticamente significativas en los tres niveles de la variable importancia de la pregunta (Figura 1). En el grupo de preguntas poco importantes, se hallaron diferencias significativas entre jóvenes y mayores, Bonferroni $=-1.865, p=0.001$, $\mathrm{y}$ entre adultos y mayores, Bonferroni $=-1.098$, $p=0.007$. En el grupo de preguntas importantes, se observaron diferencias significativas entre jóvenes y adultos, Bonferroni $=1.477, p=0.001$, entre jóvenes y mayores, Bonferroni $=2.863, p=0.001$, y entre adultos y mayores, Bonferroni $=1.386$, $p=0.001$. Finalmente, en el grupo de preguntas muy importantes, únicamente se obtuvieron diferencias significativas entre jóvenes y mayores, Bonferroni $=-1.047, p=0.004$.

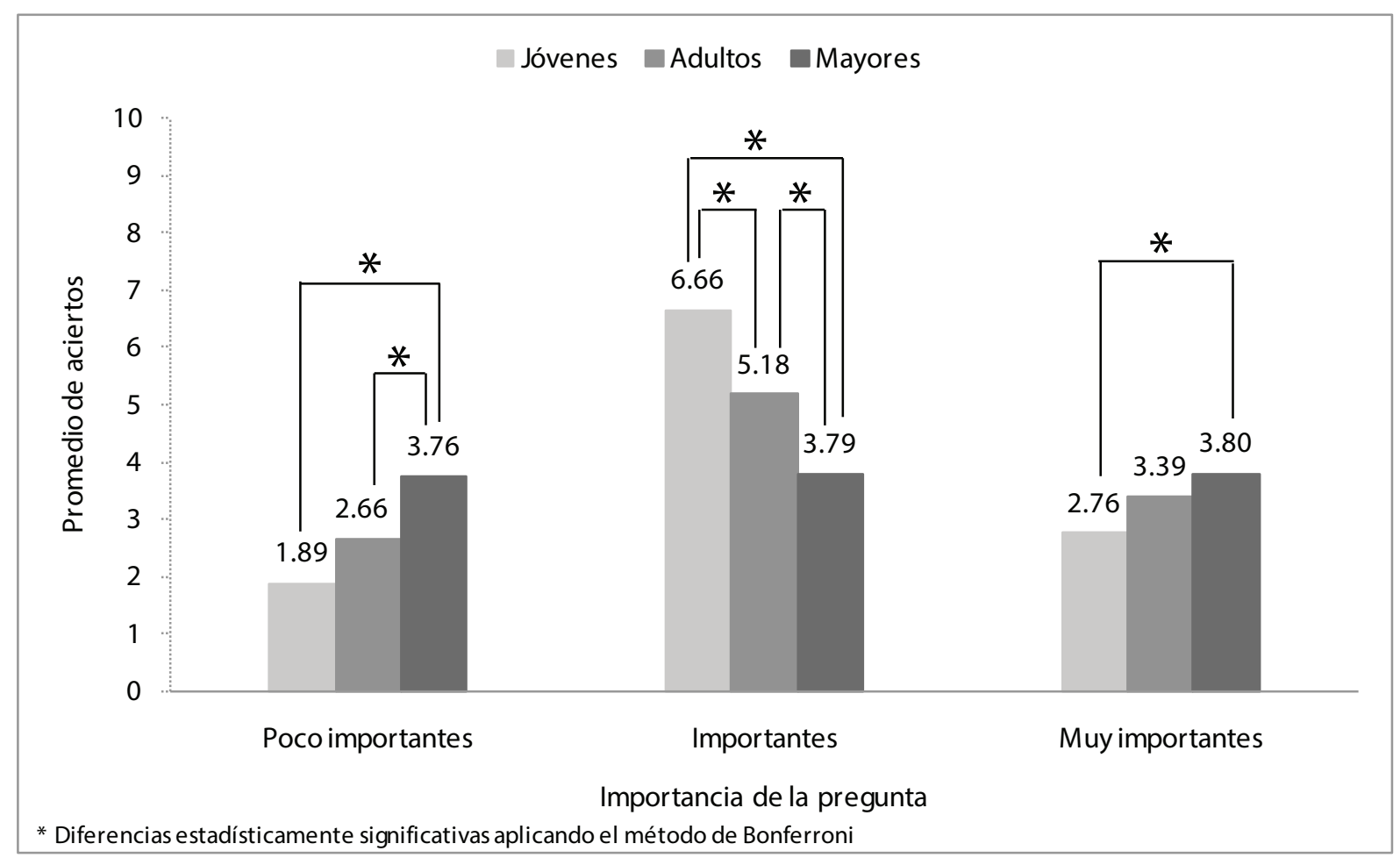

Figura 1. Media de aciertos en función del grado de importancia de la pregunta a través de los distintos grupos de edad. Fuente: elaboración propia. 


\section{Discusión}

El objetivo principal del presente estudio fue analizar el efecto de la edad en el conocimiento de la normativa de tráfico, teniendo en cuenta la importancia que tenían las preguntas para la seguridad vial. Se estableció como hipótesis que el conocimiento debía ser, en general, bajo o moderado para todos los grupos de edad. Además, de acuerdo con la literatura previa, se esperaba que los conductores mayores mostraran un conocimiento de la normativa menor que los conductores de otros grupos de edad, como consecuencia de su mayor deterioro cognitivo y de los cambios de la normativa que se produjeron con los años. Sin embargo, los resultados obtenidos son solo parcialmente consistentes con estas predicciones, debido a que los conductores mayores no siempre obtuvieron un peor rendimiento que los conductores más jóvenes. El conocimiento que manifiestan los conductores mayores aventaja al de los conductores más jóvenes, cuando se les pregunta por cuestiones consideradas poco importantes y también muy importantes para la seguridad en la conducción, siendo únicamente inferior en la categoría intermedia (importantes). Estos resultados sugieren una disociación del rendimiento en una prueba de conocimientos sobre la normativa de tráfico en función de la edad y de la importancia de las preguntas para la seguridad vial, lo que podría ser considerado como novedoso en la literatura sobre esta cuestión.

En general, el conocimiento de la normativa de tráfico que muestran los conductores de todos los grupos de edad fue pobre, en las preguntas categorizadas como poco importantes y muy importantes. Los resultados generales mejoraban ligeramente en el grupo de preguntas importantes, al menos para los conductores jóvenes y adultos (quienes mostraban una puntuación superior a 5 puntos en una escala de 0 a 10). Sin embargo, este incremento en el promedio de respuestas correctas no se apreciaba en el grupo de mayores, que mantenía un nivel de aciertos similar a los obtenidos en los otros grupos de preguntas. Estos resultados son consistentes con los obtenidos por otros autores quienes observaron que el nivel de conocimientos de distintos aspectos de la normativa era bajo o moderado para el conjunto de conductores (Buela-Casal \& Miró, 1994; Montoro \& Roca, 2007; Montoro, Roca \& Lucas, 2008; Montoro, Roca \& Tortosa, 2008).

En el caso concreto de los mayores, se observó que el conocimiento que tienen estos conductores de la normativa de tráfico era bajo, no superando la media de cuatro aciertos en ninguna de las categorías de importancia. Estos resultados pueden ser debidos al deterioro cognitivo producido por la edad, concretamente, al déficit para acceder a la memoria episódica o manejar información en la memoria operativa. Esta desventaja podría incrementarse en escenarios no familiares o ante normativas de tráfico más recientes (Caserta \& Abrams, 2007). Sin embargo, es importante destacar que la hipótesis del peor rendimiento de los conductores mayores frente a los más jóvenes solo se confirmó parcialmente, en la categoría de preguntas importantes. En los grupos de preguntas poco importantes y muy importantes, el patrón de resultados se invierte y los jóvenes muestran un peor conocimiento de la normativa que los mayores, siendo también inferior el recuerdo de los conductores adultos en la categoría de poco importantes. Más específicamente, estos resultados son consistentes, solo parcialmente, con los obtenidos por Buela-Casal y Miró (1994) quienes encontraron una relación negativa entre la antigüedad del permiso de conducir y el conocimiento de la normativa. Estos autores usaron un breve cuestionario donde se obtenía una puntación global sin diferenciar por su nivel de importancia en la seguridad vial. Además, ese estudio consideró a todos los conductores de más de 20 años de antigüedad en la misma categoría, lo que no permite separar adecuadamente el conocimiento de la normativa de conductores de mediana edad (adultos) y de mayor edad (mayores). En el presente estudio se obtuvieron resultados consistentes con las conclusiones de Buela-Casal y Miró, pero únicamente en la categoría de preguntas importantes, donde los mayores mostraban un peor recuerdo de la normativa de tráfico. Este patrón 
de respuestas era diferente del obtenido para las categorías de preguntas poco importantes y muy importantes, donde los mayores aventajaban a los conductores más jóvenes. En realidad, este patrón de respuestas resultó consistente con la observación de Buela-Casal y Miró de una ligera mejora en el recuerdo de la normativa en los conductores con mayor antigüedad en la conducción (más de 20 años), lo que los autores atribuyeron a una mayor proporción de conductores profesionales en este grupo.

Los peores resultados obtenidos por los conductores mayores en la categoría de preguntas importantes son consistentes con la hipótesis del deterioro en el rendimiento con la edad. Sin embargo, ipor qué este patrón no se mantiene con las preguntas poco y muy importantes? Una explicación tentativa de que los grupos con menor edad (y experiencia) tuvieran peores resultados, podría ser que las preguntas de las categorías poco y muy importantes incluyesen situaciones de conducción menos frecuentes. El criterio que emplearon los jueces para categorizar el nivel de importancia en el presente estudio fue el rol que cada cuestión preguntada tenía para la seguridad vial, sin tener en cuenta su frecuencia. Por ello, es posible que los jueces hayan considerado en el grupo de preguntas importantes determinadas cuestiones relevantes para la seguridad con una ocurrencia más alta en comparación con las otras dos categorías. Frente a ello, las categorías poco importantes y muy importantes podrían estar representadas por cuestiones cuya ocurrencia es más baja durante la conducción, ya sean poco relevantes (por ejemplo, "si utilizar octanaje inferior al establecido por el fabricante es aconsejable como medida de ahorro") o de gran relevancia en la seguridad vial (por ejemplo, "si se debe abrigar a un herido en un accidente"). En consecuencia, el conocimiento de las cuestiones más frecuentes (importantes) debería ser elevado para todos los conductores, pero los mayores obtienen peores resultados debido a su deterioro cognitivo. En cambio, el conocimiento de las cuestiones menos frecuentes (poco y muy importantes) debería ser bajo para todos los conductores. Sin embargo, en esta ocasión los mayores aventajan a los más jóvenes debido a su mayor experiencia en la conducción, lo que incrementa la probabilidad de experimentar situaciones poco frecuentes, facilitándoles un recuerdo mayor de dichos escenarios, frente a conductores con menos experiencia. La experiencia debería permitir recordar mejor una situación de tráfico en comparación con los que tienen menor experiencia, pues un conocimiento mayor y más diferenciado de las situaciones de tráfico les permitiría identificar la información relevante más fácilmente y codificarla de forma más rápida y fiable. Esta ventaja se podría mantener siempre que se trate de escenarios familiares o normativas de tráfico conocidas a las que se puede acceder a través de la memoria episódica (Caserta \& Abrams, 2007; Krems \& Baumann, 2009). Por lo tanto, sería recomendable en nuevos estudios examinar si la frecuencia de ocurrencia de las preguntas que conforman el cuestionario que valora el conocimiento de la normativa, se relaciona con los patrones encontrados en función de la importancia que tengan para la seguridad vial.

Los resultados obtenidos nos llevan a plantear una reflexión enjuiciando la problemática que se deriva, en general, de los resultados de esta investigación y de otras realizadas recientemente (Montoro \& Roca, 2007; Montoro, Roca \& Lucas, 2008; Montoro, Roca \& Tortosa, 2008), sobre el relativo desconocimiento de las normas de tráfico por el conjunto de conductores. Un buen conocimiento de las mismas se asocia con una disminución del número de fallecimientos (Evans, 1991). En consecuencia, se podrían proponer medidas para facilitar el recuerdo de normas consideras relevantes para la seguridad vial donde se incluyeran también los cambios normativos más recientes. Para ello, se podrían incrementar las campañas en medios de comunicación, trípticos en estaciones de servicio u otros lugares donde se pueda acceder con facilidad a la población conductora. En el caso de conductores mayores, estas medidas podrían ser reforzadas con cursos que mejoraran los posibles déficits cognitivos (como en memoria operativa y episódica) producidos por la edad, favoreciendo que se mantenga un adecua- 
do conocimiento de las normas de circulación y una mejor capacidad para afrontar situaciones dinámicas de tráfico.

\section{Referencias}

Barrash, J., Stillman, A., Anderson, S. W., Uc, E. Y., Dawson, J. D. \& Rizzo M. (2010). Prediction of driving ability with neuropsychological tests: Demographic adjustments diminish accuracy. Journal of the International Neuropsychological Society, 16(4), 679-686.

Buela-Casal, G. \& Miró, E. (1994). Conocimiento y actitudes hacia el Código de Circulación y regulación de la conducta. Mapfre Seguridad, 54, 11-19.

Bédard, M., Guyatt, G. H., Stones, M. J. \& Hirdes, J. P. (2002). The independent contribution of drive, crash and vehicle characteristics to driver fatalities. Accident Analysis and Prevention, 34(6), 717-727.

Campagne, A., Pebayle, T. \& Muzet, A. (2004). Correlation between driving errors and vigilance level: Influence of the driver's age. Physiology and Behavior, 80(4), 515-524.

Caserta, R. J. \& Abrams, L. (2007). The relevance of situation awareness in older adults' cognitive functioning: A review. European Review of Aging and Physical Activity, 4(1), 3-13.

Charlton, J., Oxley, J., Scully, J., Koppel, S., Congiu, M., Muir, C., et al. (2006). Self-regulatory driving practices of older drivers in the Australian Capital Territory and New South Wales. Clayton, Australia: Monash University Accident Research Centre.

Eby, D. W., Trombley, D. A., Molnar, L. J. \& Shope, J. T. (1998). The assessment of older driver' capabilities: A review of the literature. Michigan, MI: The University of Michigan Transportation Research Institute.

Evans, L. (1991). Traffic safety and the driver. New York, NY: Van Nostrand Reinhold.

Hakamies-Blomqvist, L. (1998). Older drivers' accident risk: Conceptual and methodological issues. Accident Analysis and Prevention, 30(3), 293-297.

Instituto de Mayores y Servicios Sociales. (2009). Las personas mayores en España. Madrid, España: Ministerio de Sanidad y Política Social.
Insurance Institute for Highway Safety. (2001). Older drivers [Special issue]. Status Report, 36, 1-7.

Krems, J. F. \& Baumann, M. R. K. (2009). Driving and situation awareness: A cognitive model of memory-update processes. En M. Kurosu (Ed.), Human Centered Design (pp. 986-994). Berlin, Alemania: Springer.

Li, G., Braver, E. R. \& Chen, L. H. (2003). Fragility versus excessive crash involvement as determinants of high death rates per vehicle-mile of travel among older drivers. Accident Analysis and Prevention, 35(2), 227-235.

Lundberg, C., Hakamies-Blomqvist, L., Almkvist, O. \& Johansson, K. (1998). Impairments of some cognitive functions are common in crash-involved older drivers. Accident Analysis and Prevention, 30(3), 371-377.

Mathias, J. L. \& Lucas, L. K. (2009). Cognitive predictors of unsafe driving in older drivers: A meta-analysis. International Psychogeriatrics, 21(4), 637-653.

Marmeleira, J., Godinho, M. \& Vogelaere, P. (2009). The potential role of physical activity on driving performance and safety among older adults. European Review of Aging and Physical Activity, 6(1), 29-38.

Montoro, L. \& Roca, J. (2007). El permiso por puntos: percepción de los conductores. Barcelona, España: ARAG - Compañía Internacional de Seguros y Reaseguros, S.A.

Montoro, L., Roca, J. \& Lucas, A. (2008). Estudio ARAG 2008: La velocidad en el tráfico. (Informe de investigación). Barcelona, España: ARAG - Compañía Internacional de Seguros y Reaseguros, S.A. Montoro, L., Roca, J. \& Tortosa, F. (2008). Influencia del permiso de conducción por puntos en el comportamiento al volante: percepción de los conductores. Psicothema, 20(4), 652-658.

Oxley, J., Charlton, J., Fildes, B., Koppel, S., Scully, J., Congiu, M., et al. (2005). Crash risk of older female drivers. Clayton, Australia: Monash University Accident Research Centre.

Prada, E. L., Pineda, G. E., Mejía, M. A. \& Conde, C. A. (2010). Prueba computarizada Memonum: efecto de intervalos y distractores sobre la memoria de 
trabajo en mujeres mayores de 50 años. Universitas Psychologica, 9(3), 893-905.

Simões, A. \& Marin-Lamellet, C. (2002). Road users who are elderly: Drivers and pedestrians. En R. Fuller \& J. A. Santos (Eds.), Human factors for highway engineers (pp. 255-275). Amsterdam, Holanda: Pergamon. 


\section{Apéndice 1}

Cuestionario de conocimientos sobre la normativa de tráfico

\section{a) Preguntas poco importantes de acuerdo con la valoración de 6 jueces expertos.}

1. Si la batería de su turismo necesita un mantenimiento periódico, ¿qué debe hacer si el nivel de líquido de la misma es bajo?

a. Añadir una disolución de agua destilada y ácido sulfúrico, en iguales proporciones.

b. Añadir agua destilada, únicamente.

c. Añadir una mezcla de líquidos recomendada por el fabricante, según el tipo y marca de la batería.

2. Al repostar gasolina en su vehículo, usted ha llenado el depósito con gasolina de octanaje inferior al establecido por el fabricante, ies aconsejable como medida de ahorro?

a. Sí, porque la emisión de humos es menor.

b. No, porque se daña el motor.

c. Sí, puesto que, aunque disminuye la potencia del motor, su precio es más bajo.

3. La obtención del permiso de la clase B, iimplica la concesión de otros permisos?

a. No.

b. Sí, los permisos de las clases A1 y A.

c. Sí, el de la clase E.

4. En un carril reservado para autobuses, ile está permitido parar su turismo?

a. No, como norma general.

b. Sí, siempre que deje espacio para que puedan pasar los autobuses, y que la línea que separa el carril sea discontinua.

c. Sólo cuando sea por tiempo inferior a 2 minutos.

5. Como normal general, iestá prohibido parar o estacionar su vehículo en doble fila cuando no exista un lugar adecuado en las proximidades?

a. Sí, está prohibido parar y estacionar.

b. Estacionar sí, pero no parar.

c. No salvo que se impida el paso de otros vehículos o se trate de una calzada estrecha.

6. Si con su turismo arrastra un remolque de 750 kilogramos de masa máxima autorizada, idebe llevar una tarjeta de inspección técnica del remolque?

a. No, porque solo es obligatorio si el remolque supera los 750 kilogramos de masa máxima autorizada.

b. Sí, además, en la tarjeta de inspección técnica del turismo deberá figurar que este lleva un sistema de acoplamiento compatible con el del remolque.

c. No, es suficiente con llevar la tarjeta de inspección técnica del turismo, siempre que en su reverso figure que éste lleva un sistema de acoplamiento compatible.

7. Si necesariamente debe circular por un tramo de calzada anegado de agua, icómo debe hacerlo?

a. En primera, lentamente y a una velocidad y aceleración sostenida procurando que la velocidad del motor y la relación de marchas estén adecuadas.

b. Acelerando, para atravesar la zona lo antes posible.

c. Pisando ligera y suavemente el pedal del freno de forma continuada hasta que se haya atravesado la zona inundada.

8. El seguro de Responsabilidad Civil de suscripción obligatoria, vulgarmente denominado "seguro obligatorio", icubre los daños en la persona del conductor del vehículo asegurado?

a. Sí, siempre. 
b. No.

c. Sí, pero únicamente cuando el conductor no es propietario del vehículo.

9. ¿Cree Ud. que si un vehículo tiene un ralentí demasiado alto influye en el consumo de carburante?

a. Sí, porque al girar el motor más deprisa, consume más.

b. Sí, porque consume menos carburante.

c. No, tal circunstancia no influye ni altera el consumo.

10. Los padres responderán solidariamente ante una infracción de tráfico cometida por un menor. Y esta responsabilidad tiene carácter:

a. Penal.

b. Pecuniario.

c. Civil.

\section{b) Preguntas importantes de acuerdo con la valoración de 6 jueces expertos.}

1. Usted ha adelantado con su turismo en una autopista a otro turismo que circula por el carril derecho. Si dicho carril está libre y no desea realizar otro adelantamiento, idebe regresar al carril derecho?

a. Sí.

b. Solo cuando la calzada tenga dos carriles.

c. Solo cuando moleste a los vehículos que circulen detrás a mayor velocidad.

2. En los tramos de vía en los que por su estrechez es imposible o muy difícil el paso simultáneo de dos vehículos que circulen en sentido contrario, ¿qué vehículo tendrá preferencia de paso si no hay señalización expresa?

a. El vehículo de mayor peso máximo autorizado.

b. El vehículo que haya entrado primero.

c. El vehículo de mayor longitud.

3. Como norma general, las advertencias que los conductores deben realizar para advertir al resto de los usuarios de las maniobras que vayan a efectuar con sus vehículos, se harán...

a. Utilizando la señalización luminosa del vehículo o, en su defecto, con el brazo.

b. Utilizando únicamente la señalización luminosa.

c. Utilizando simultáneamente la señalización luminosa y acústica.

4. Como norma general, iestá permitido adelantar en los pasos de peatones?

a. Sí.

b. No.

5. La luz amarilla fija de un semáforo, ¿le obliga siempre a detenerse?

a. Sí, siempre.

b. Sí, salvo que, cuando se encienda, se encuentre el vehículo tan cerca del lugar de detención que no pueda detenerse antes del mismo en condiciones de seguridad suficiente.

c. Solo si en el mismo poste del semáforo hay una señal de STOP.

6. Con su turismo se aproxima a una intersección sin señalizar en la que no goza de prioridad de paso, ia qué velocidad debe circular?

a. A una velocidad no superior a 50 kilómetros por hora.

b. A una velocidad moderada que, en caso de necesidad, me permita detener el vehículo para cumplir las normas de prioridad.

c. A una velocidad no inferior a la mínima establecida para la vía.

7. Con su turismo, ile está permitido remolcar a otro turismo accidentado o averiado?

a. Sí, pero solo en autopistas o autovías. 
b. No, en ningún caso.

c. Solo excepcionalmente, y con las debidas condiciones de seguridad, hasta el lugar más próximo donde pueda quedar convenientemente inmovilizado y sin entorpecer la circulación, siempre que no se trate de autopistas o autovías.

8. En poblado, si el turismo que Ud. conduce dispone de cinturones de seguridad en todos los asientos, ¿es obligatorio el uso de dicho cinturón por el conductor y los ocupantes del mismo?

a. Solo es obligatorio para el conductor.

b. Solo es obligatorio para el conductor y para los ocupantes de los asientos delanteros.

c. Sí, es obligatorio tanto para el conductor como para el resto de los ocupantes.

9. Esta señal, iqué le indica?

a. Que circulo por una calzada en la que debo de ceder el paso a los vehículos que se aproximen por mi derecha en las intersecciones.

b. Que circulo por una calzada que tiene prioridad de paso en las intersecciones sobre los vehículos que circulen por otra calzada o procedan de ella.

c. Que circulo por una vía pública calificada de atención preferente.

10. A un vehículo de dos ruedas se le puede adelantar en una intersección o próximo a ella...

a. Si, si la calzada goza de prioridad y esta señalizada.

b. Sí.

c. No.

\section{c) Preguntas muy importantes de acuerdo con la valoración de 6 jueces expertos.}

1. En una intersección de una vía pavimentada con otra sin pavimentar, ¿qué vehículos tienen preferencia?

a. Los vehículos que se aproximen por la derecha.

b. Los vehículos que circulen por la vía pavimentada.

c. Los vehículos que circulen por la vía más ancha.

2. Con su turismo se aproxima a una intersección en la que existe un semáforo encendido con luz amarilla intermitente. ¿Debe ceder el paso a un vehículo que se aproxime a la intersección por la derecha?

a. Sí, porque el semáforo me obliga a cumplir la regla general de prioridad de paso.

b. Sí, pero únicamente cuando junto al semáforo haya una señal que me obligue a ceder el paso.

c. No, porque el semáforo me indica que debo circular con precaución pero no obliga a ceder el paso.

3. En autopista, ile está permitido hacer un cambio de sentido de la marcha utilizando los pasos de la mediana que separa ambos sentidos de circulación si las circunstancias de tráfico lo permiten?

a. Sí, siempre que no exista obstáculo que lo impida.

b. No, porque está prohibido.

c. Sí, pero únicamente para salir de la autopista.

4. A los heridos en un accidente de circulación, hasta que llegue la correspondiente ayuda, se les debe abrigar...

a. Pero solo a los muy graves que tengan hemorragias.

b. Pero solo cuando haga frío.

c. Como norma general, tanto en invierno como en verano, para evitar la pérdida de calor.

5. Un turismo, ipuede remolcar a otro turismo que está averiado y no puede ser arrastrado por otro vehículo destinado a ese fin?

a. Sí, pero solo cuando el conductor del turismo que va a realizar el remolque posee el permiso B+E.

b. No, porque está siempre prohibido el remolque por un vehículo distinto al de un vehículo grúa. 
c. Sí, solo se podrá realizar hasta el lugar más próximo donde pueda quedar convenientemente inmovilizado sin entorpecer la circulación y siempre que no se circule por autopista o autovía.

6. En las proximidades de una curva de visibilidad reducida, está prohibido...

a. Parar y estacionar.

b. Parar, únicamente.

c. Estacionar, únicamente.

7. En una curva sin visibilidad en la que los dos sentidos están claramente separados por una línea continua, iestá permitido adelantar a un ciclomotor?

a. No, en ningún caso.

b. Sí, aunque para ello sea necesario invadir la parte izquierda de la calzada dada la reducida velocidad a la que circula.

c. Sí, pero siempre que la maniobra pueda efectuarse con seguridad, sin invadir la zona reservada al sentido contrario y guardando la distancia lateral reglamentaria.

8. Una norma fundamental, si tenemos que atender a heridos en un accidente de circulación, es...

a. Darles alguna bebida estimulante para reanimarlos.

b. Trasladarlos en nuestro vehículo con la mayor urgencia posible.

c. Procurar moverlos lo menos posible.

9. Si en su turismo lleva como pasajero a un niño que tiene 5 años, ien qué asiento debe situarlo si no dispone de un asiento de seguridad u otro dispositivo concebido específicamente y homologado para menores?

a. En los asientos traseros.

b. En el asiento delantero.

c. En los asientos delanteros o traseros, indistintamente.

10. Cuando se vaya a girar el vehículo para entrar en una vía y haya peatones cruzándola, y no exista paso de peatones, tiene la preferencia...

a. El peatón.

b. El vehículo.

c. El peatón, solo si es mayor, niño o discapacitado. 
